\title{
Integrative analysis of key candidate genes and signaling pathways in ovarian cancer by bioinformatics
}

\author{
Cuicui Dong ${ }^{1 \dagger}$, Xin Tian ${ }^{1 \dagger}$, Fucheng He${ }^{2}$, Jiayi Zhang ${ }^{1}$, Xiaojian Cui ${ }^{1}$, Qin He ${ }^{1}$, Ping Si ${ }^{{ }^{*}}$ and Yongming Shen ${ }^{1 *}$
}

\begin{abstract}
Background: Ovarian cancer is one of the most common gynecological tumors, and among gynecological tumors, its incidence and mortality rates are fairly high. However, the pathogenesis of ovarian cancer is not clear. The present study aimed to investigate the differentially expressed genes and signaling pathways associated with ovarian cancer by bioinformatics analysis.

Methods: The data from three mRNA expression profiling microarrays (GSE14407, GSE29450, and GSE54388) were obtained from the Gene Expression Omnibus (GEO) database. Differentially expressed genes between ovarian cancer tissues and normal tissues were identified using $\mathrm{R}$ software. The overlapping genes from the three GEO datasets were identified, and profound analysis was performed. The overlapping genes were used for pathway and Gene Ontology (GO) functional enrichment analysis using the Metascape online tool. Protein-protein interactions were analyzed with the Search Tool for the Retrieval of Interacting Genes/Proteins (STRING). Subnetwork models were selected using the plugin molecular complex detection (MCODE) application in Cytoscape. Kaplan-Meier curves were used to analyze the univariate survival outcomes of the hub genes. The Human Protein Atlas (HPA) database and Gene Expression Profiling Interactive Analysis (GEPIA) were used to validate hub genes.
\end{abstract}

Results: In total, 708 overlapping genes were identified through analyses of the three microarray datasets (GSE14407, GSE29450, and GSE54388). These genes mainly participated in mitotic sister chromatid segregation, regulation of chromosome segregation and regulation of the cell cycle process. High CCNA2 expression was associated with poor overall survival (OS) and tumor stage. The expression of CDK1, CDC20, CCNB1, BUB1B, CCNA2, KIF11, CDCA8, KIF2C, NDC80 and TOP2A was increased in ovarian cancer tissues compared with normal tissues according to the Oncomine database. Higher expression levels of these seven candidate genes in ovarian cancer tissues compared with normal tissues were observed by GEPIA. The protein expression levels of CCNA2, CCNB1, CDC20, CDCA8, CDK1, KIF11 and TOP2A were high in ovarian cancer tissues, which was further confirmed via the HPA database.

Conclusion: Taken together, our study provided evidence concerning the altered expression of genes in ovarian cancer tissues compared with normal tissues. In vivo and in vitro experiments are required to verify the results of the present study.

\footnotetext{
${ }^{*}$ Correspondence: kangsiping@live.cn; shenym2020@sina.com

${ }^{\dagger}$ Cuicui Dong and Xin Tian contributed equally to this article.

${ }^{\dagger}$ Cuicui Dong and Xin Tian should be listed as co-first authors.

1 Department of Clinical Lab, The Children's Hospital of Tianjin (Children's

Hospital of Tianjin University), No. 238, Longyan Road, Beichen District,

Tianjin 300000, PR China

Full list of author information is available at the end of the article
} original author(s) and the source, provide a link to the Creative Commons licence, and indicate if changes were made. The images or other third party material in this article are included in the article's Creative Commons licence, unless indicated otherwise in a credit line to the material. If material is not included in the article's Creative Commons licence and your intended use is not permitted by statutory regulation or exceeds the permitted use, you will need to obtain permission directly from the copyright holder. To view a copy of this licence, visit http://creativecommons.org/licenses/by/4.0/. The Creative Commons Public Domain Dedication waiver (http://creativeco mmons.org/publicdomain/zero/1.0/) applies to the data made available in this article, unless otherwise stated in a credit line to the data. 
Keywords: Ovarian Cancer, Gene Expression Omnibus, Bioinformatics Analysis, Hub Genes

\section{Introduction}

Ovarian cancer is one of the most common gynecological tumors associated with poor survival in women worldwide $[1,2]$. The majority of newly diagnosed ovarian cancer patients are treated with radical surgery, followed by six to eight cycles of adjuvant platinum and taxane combination chemotherapy [3]. Three or more cycles of neoadjuvant chemotherapy prior to debulking surgery and adjuvant chemotherapy are alternative options for some patients [3]. However, the 5-year survival rate of ovarian cancer patients is approximately $30 \%$ [4]. Therefore, it is crucial to understand the pathogenesis of ovarian cancer. Next-generation sequencing (NGS) technology has become widely available and is used to determine a patient's precise genetic profile and to identify novel mutations to serve as new drug targets [5]. Furthermore, NGS-based mutation panels profile multiple genes simultaneously, allowing for the reporting of numerous genes while saving labor and resources [5]. Gene expression microarrays have been used in many studies to identify differentially expressed genes (DEGs) and pathways associated with ovarian cancer.

A comprehensive analysis of the interactions between DEGs and enriched pathways will contribute to the understanding of the physiopathology of ovarian cancer progression and tumorigenesis. With the increase in the number of publications about GEO datasets, GEO has become a potential resource for bioinformatics studies.

In this study, we downloaded three datasets (GSE14407, GSE29450, and GSE54388) from the Gene Expression Omnibus (GEO) (http://www.ncbi.nlm.nih.gov/geo) database. Furthermore, $\mathrm{R}$ software with the limma package was used to identify DEGs.

Moreover, a Venn diagram was generated to identify DEGs between ovarian cancer tissues and normal tissues that overlapped in the three GEO profiles. Subsequently, we conducted functional enrichment analyses and established a protein-protein interaction (PPI) network. Kaplan-Meier plotter, the Human Protein Atlas (HPA) database, the Oncomine database and Gene Expression Profiling Interactive Analysis (GEPIA) were used to validate the selected hub genes. In conclusion, the current research based on bioinformatics was designed to explore some potential molecular biomarkers of ovarian cancer.

\section{Material and methods}

Ethics committee or institutional review board approval was not needed for this study, as all the data originated from a public database.

\section{Differentially expressed genes analysis}

Three gene expression profiles (GSE54388, GSE14407, and GSE29450) were downloaded from the GEO database. GSE54388 contains 6 human ovarian surface epithelium samples and 16 tumor epithelial component samples. GSE14407 contains 12 healthy ovarian surface epithelium samples and 12 serous ovarian cancer epithelium samples. GSE29450 contains 10 ovarian cancer cell specimens and 10 normal ovarian surface epithelium specimens. The GSE54388, GSE14407, and GSE29450 data were all obtained with the Affymetrix Human Genome U133 Plus 2.0 Array platform. Expression data were normalized using median normalization [6]. DEGs between ovarian carcinoma tissues and normal tissues were obtained by using R statistical software and the limma Bioconductor package [7]. The criteria for DEG screening were FDR adjusted $\mathrm{P}<0.05$ and $|\log \mathrm{FC}| \geq 1$. Volcano plots and heatmaps were generated with $\mathrm{R}$ (Bioconductor, Roswell Park Cancer Institute, Buffalo NY, USA).

\section{Functional enrichment analyses of overlapping DEGs}

We used the online software Venn diagram (http:// www.bioinformatics.com.cn/static/others/jvenn/examp le.html) to identify the overlapping genes in the $3 \mathrm{GEO}$ datasets [8]. These common genes were submitted to the Metascape online tool [9] and used for Gene Ontology (GO) and Kyoto Encyclopedia of Genes and Genomes (KEGG) analyses. Thresholds were set to $P$-value $<0.05$, minimum count of 3 , and enrichment factor of $>1.5$. The top 20 GO and KEGG terms were selected and are shown in the graphs.

\section{PPI network}

These common genes were submitted to the Search Tool for the Retrieval of Interacting Genes (STRING; http://string-db.org) (version 11.0) and visualized using Cytoscape. The minimum required interaction score was set to 0.9 , and disconnected nodes in the network were hidden. Then, we used the MCODE app of Cytoscape to indentify the significant modules in the PPI network (maximum depth $=100$, node score cutoff $=0.2$, degree cutoff $=2$, and $\mathrm{k}$-core $=2$ ) [10]. The top five MCODE models are listed, and the top ten hub genes were used for further analyses.

\section{Validation of the hub genes}

The expression levels of the selected hub genes was further analyzed using the Oncomine database (http:// www.oncomine.org) and GEPIA database (http:// 
gepia.cancer-pku.cn/). Boxplots, stage plots and survival analyses for these hub genes were further performed using the GEPIA database (http://gepia. cancer-pku.cn/).

Moreover, the HPA database (http://www.proteinatlas. org/) was searched and retrieved to compare the protein expression of hub genes in cancerous specimens with that in normal specimens. The HPA database, comprising more than 10 million pictures showing human protein expression patterns in tissues and cells, is publicly available to allow researchers to freely study the human proteome [11].

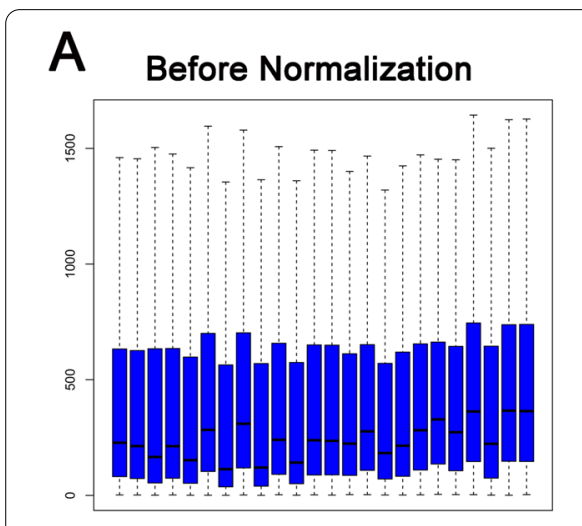

C

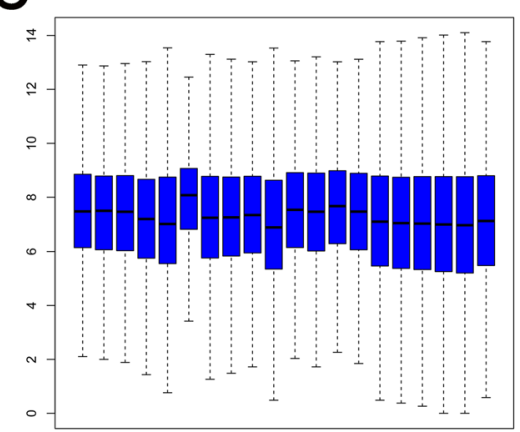

\section{E Before Normalization}

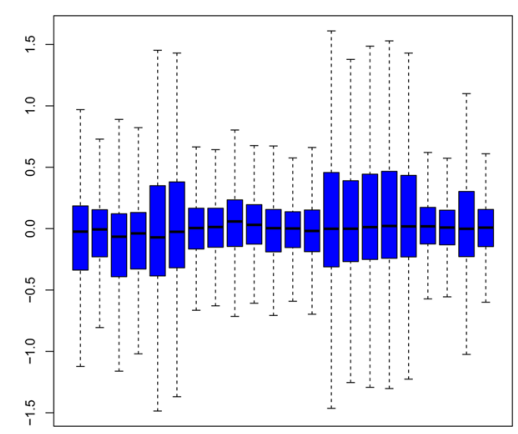

After Normalization

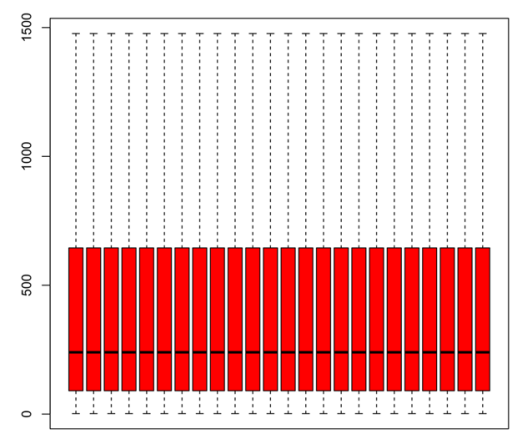

After Normalization

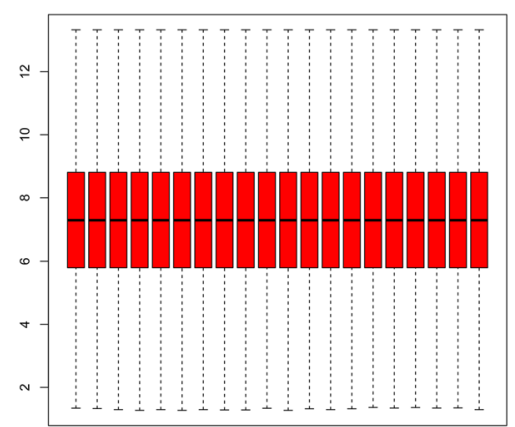

After Normalization

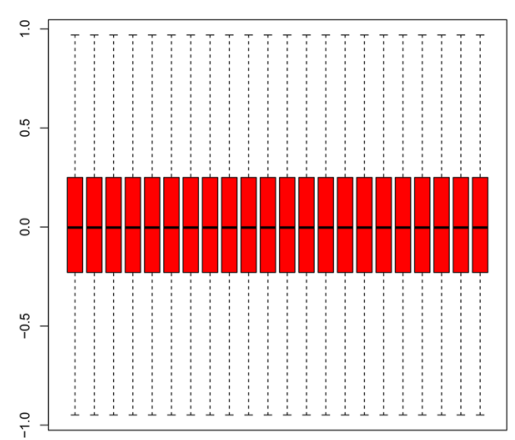

B
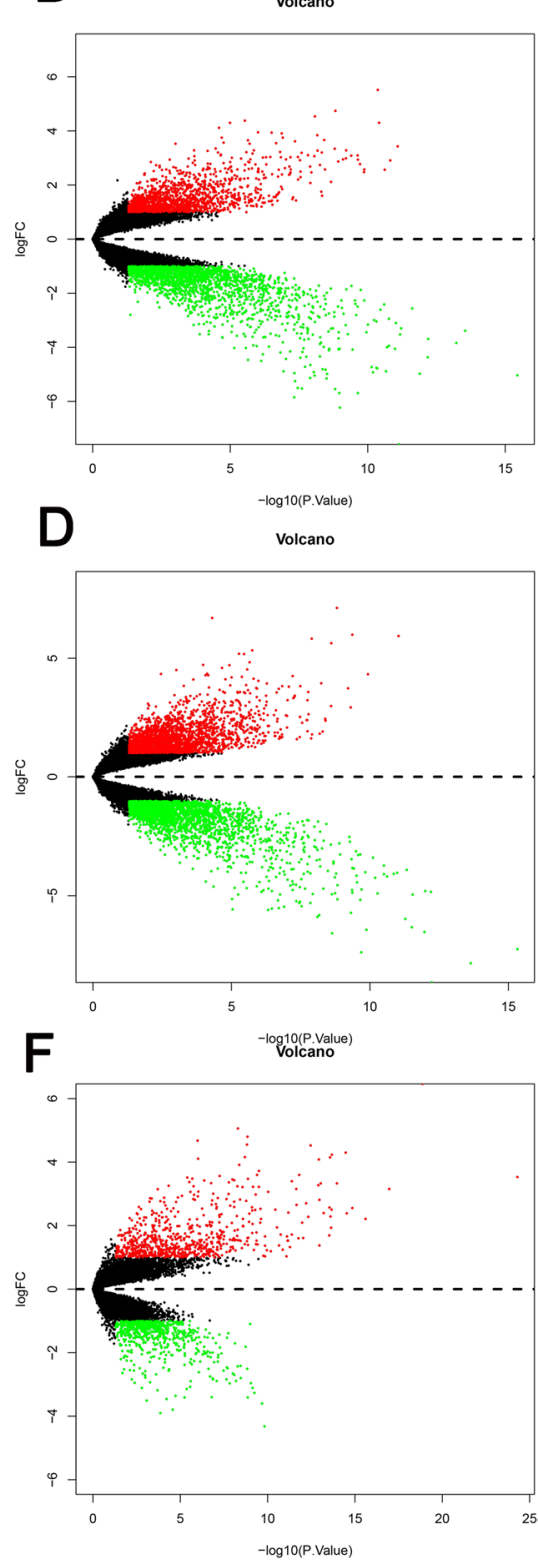

Fig. 1 Differentially expressed genes in GSE54388, GSE14407, and GSE29450. A Box plot comparing the distribution of the expression values for all the samples after normalization in GSE54388. B Volcano plot of differentially expressed genes (DEGs) in GSE29450. Green dots represent downregulated genes and red dots represent upregulated genes in breast cancer tissue; black dots represent normally expressed genes. $\mathbf{C}$ Box plot comparing the distribution of the expression values for all the samples after normalization in GSE14407. D Volcano plot of differentially expressed genes (DEGs) in GSE14407. Green dots represent downregulated genes and red dots represent upregulated genes in breast cancer tissue; black dots represent normally expressed genes. E Box plot comparing the distribution of the expression values for all the samples after normalization in GSE29450. F Volcano plot of differentially expressed genes (DEGs) in GSE29450. Green dots represent downregulated genes and red dots represent upregulated genes in breast cancer tissue; black dots represent normally expressed genes 
Table 1 Top ten differentially expressed genes in ovarian cancer than normal tissue in GSE14407

\begin{tabular}{|c|c|c|c|c|c|c|}
\hline Gene & $\log \mathrm{FC}$ & AveExpr & $t$ & $P$ Value & adj.P.Val & B \\
\hline NELL2 & -5.0322 & 11.33906 & -17.8843 & 3.62E-16 & $7.84 \mathrm{E}-12$ & 26.13203 \\
\hline TXNIP & -3.38158 & 11.92188 & -14.8911 & $2.85 \mathrm{E}-14$ & $3.09 \mathrm{E}-10$ & 22.23984 \\
\hline ARHGAP18 & -3.83976 & 11.43925 & -14.4286 & 5.96E-14 & 4.30E-10 & 21.56643 \\
\hline RERG & -3.69045 & 9.294558 & -13.0174 & $6.38 \mathrm{E}-13$ & 2.85E-09 & 19.37512 \\
\hline AQP9 & -4.36576 & 9.957475 & -12.9985 & $6.59 \mathrm{E}-13$ & 2.85E-09 & 19.34429 \\
\hline REEP1 & -4.97147 & 9.464285 & -12.6144 & $1.30 \mathrm{E}-12$ & 4.69E-09 & 18.70929 \\
\hline FRY & -2.55897 & 8.842311 & -12.2756 & $2.40 \mathrm{E}-12$ & 7.41E-09 & 18.13482 \\
\hline ANKRD29 & -3.29846 & 8.646326 & -11.7771 & $6.03 \mathrm{E}-12$ & $1.58 \mathrm{E}-08$ & 17.26458 \\
\hline DPYD & -3.52501 & 9.751954 & -11.7269 & $6.62 \mathrm{E}-12$ & $1.58 \mathrm{E}-08$ & 17.17536 \\
\hline ITLN1 & -7.58571 & 10.52319 & -11.6755 & 7.30E-12 & $1.58 \mathrm{E}-08$ & 17.0836 \\
\hline
\end{tabular}

\section{Results}

Identification of DEGs in ovarian cancers

First, we normalized the read counts for each sample. The median values of each sample were almost consistent, suggesting that the data were eligible for further analysis (Fig. 1 A, C and E). A total of 3486 DEGs (upregulated $=1950$; downregulated $=1536$ ) were identified in GSE14407 (Fig. 1 B), and the top ten DEGs are listed in Table 1. A total of 4780 DEGs (upregulated $=2407$; downregulated $=2373$ ) were identified in GSE29450 (Fig. 1 D), and the top ten DEGs are listed in Table 2. A total of 1368 DEGs (upregulated $=625$; downregulated $=743$ ) were identified in GSE54388 (Fig. $1 \mathrm{~F}$ ), and the top ten DEGs are listed in Table 3. Then, 708 overlapping genes were finally selected for further analysis (Fig. 2).

\section{KEGG and GO enrichment analyses}

Next, we identified enriched gene ontology and KEGG pathway annotations associated with the overlapping genes. The top twenty GO and KEGG terms were as follows (Fig. 3): mitotic sister chromatid segregation, regulation of chromosome segregation, regulation of cell cycle process, PID AURORA B Pathway, gonad development, regulation of cell division, attachment of spindle microtubules to kinetochore, NABA CORE MATRISOME, epithelial cell differentiation, extracellular structure organization, cellular response to organic cyclic compound, muscle tissue development, factors involved in megakaryocyte development and platelet production, blood vessel development, cell cycle, development growth, regulation of protein serine/threonine kinase activity, PID FOXM1 PATHWAY, cerebral cortex development, and response to inorganic substance.

\section{PPI network and significant module analysis}

We set the minimum required interaction score to 0.09 in the STRING database. There were 656 nodes and 1231 edges in this PPI network (Fig. 4). The most significant module, identified using the MCODE app in Cytoscape, is shown in Fig. 5.

We selected the top 10 hub genes (CDK1, CDC20, CCNB1, BUB1B, CCNA2, KIF11, CDCA8, KIF2C, NDC80 and TOP2A, Fig. 6) according to the joint points from the STRING database.

Table 2 Top ten differentially expressed genes in ovarian cancer than normal tissue in GSE29450

\begin{tabular}{|c|c|c|c|c|c|c|}
\hline Gene & $\log F C$ & AveExpr & $t$ & $P$ Value & adj.P.Val & B \\
\hline BNC1 & -7.25722 & 8.828002 & -21.0627 & $4.69 \mathrm{E}-16$ & $1.02 \mathrm{E}-11$ & 25.517 \\
\hline CALB2 & -7.84629 & 10.12555 & -17.457 & $2.32 \mathrm{E}-14$ & $2.51 \mathrm{E}-10$ & 22.25684 \\
\hline ITLN1 & -8.65669 & 11.21177 & -14.8908 & $5.86 \mathrm{E}-13$ & 3.38E-09 & 19.40216 \\
\hline LINC01105 & -4.83459 & 7.945648 & -14.8442 & $6.24 \mathrm{E}-13$ & 3.38E-09 & 19.34546 \\
\hline LINC00842 & -4.7977 & 5.78064 & -14.489 & $1.01 \mathrm{E}-12$ & 3.88E-09 & 18.90674 \\
\hline CLEC4M & -6.52877 & 8.317774 & -14.4461 & $1.07 \mathrm{E}-12$ & 3.88E-09 & 18.85291 \\
\hline C21 orf62 & -4.94547 & 7.809939 & -13.7446 & $2.89 \mathrm{E}-12$ & 8.39E-09 & 17.94981 \\
\hline GPR133 & -6.31894 & 7.815952 & -13.6959 & $3.10 \mathrm{E}-12$ & 8.39E-09 & 17.88546 \\
\hline PEX5L & -3.90997 & 6.84449 & -13.4133 & $4.67 \mathrm{E}-12$ & $1.12 \mathrm{E}-08$ & 17.50705 \\
\hline SERTM1 & -5.97807 & 9.900058 & -13.3287 & 5.29E-12 & $1.15 \mathrm{E}-08$ & 17.39221 \\
\hline
\end{tabular}


Table 3 Top ten differentially expressed genes in ovarian cancer than normal tissue in GSE54388

\begin{tabular}{lllllll}
\hline Gene & logFC & AveExpr & $\mathbf{t}$ & PValue & adj.P.Val & B \\
\hline LINC01105 & 3.533815 & 0.858675 & 50.96816 & $4.99 \mathrm{E}-25$ & $1.08 \mathrm{E}-20$ & 40.50396 \\
ITLN1 & 6.462358 & 1.614636 & 29.20985 & $1.37 \mathrm{E}-19$ & $1.48 \mathrm{E}-15$ & 32.75399 \\
WNT2B & 3.157712 & 0.658476 & 23.96666 & $1.09 \mathrm{E}-17$ & $7.89 \mathrm{E}-14$ & 29.30784 \\
ABCA8 & 2.207886 & 0.566936 & 20.76557 & $2.51 \mathrm{E}-16$ & $1.36 \mathrm{E}-12$ & 26.65956 \\
ADH1C & 2.551191 & 0.469443 & 19.17573 & $1.41 \mathrm{E}-15$ & $6.11 \mathrm{E}-12$ & 25.14902 \\
CLDN15 & 2.384036 & 0.47813 & 18.51044 & $3.01 \mathrm{E}-15$ & $1.07 \mathrm{E}-11$ & 24.47264 \\
MGARP & 4.30019 & 1.069266 & 18.39105 & $3.46 \mathrm{E}-15$ & $1.07 \mathrm{E}-11$ & 24.34831 \\
CALB2 & 3.326602 & 0.818659 & 17.43105 & $1.09 \mathrm{E}-14$ & $2.95 \mathrm{E}-11$ & 23.31443 \\
PRG4 & 4.229521 & 1.027993 & 16.90125 & $2.10 \mathrm{E}-14$ & $4.27 \mathrm{E}-11$ & 22.71661 \\
LHX2 & 2.50154 & 0.618326 & 16.86466 & $2.20 \mathrm{E}-14$ & $4.27 \mathrm{E}-11$ & 22.67457 \\
\hline
\end{tabular}

\section{Validation of the hub genes}

To determine differences in the expression of these ten hub genes in ovarian cancer tissues and normal tissues, the hub gene mRNA levels in ovarian cancer tissues and normal tissues were further analyzed using the Oncomine database. The results revealed that CDK1, CDC20, CCNB1, BUB1B, CCNA2, KIF11, CDCA8, KIF2C, NDC80 and TOP2A were all expressed at higher levels in ovarian cancer tissues than in normal tissues (Fig. 7 and 8).
Correlations between CDK1, CDC20, CCNB1, BUB1B, CCNA2, KIF11, CDCA8, KIF2C, NDC80 and TOP2A expression and tumor stage were further analyzed in ovarian cancer patients (GEPIA). The distribution of BUB1B and CCNA2 expression correlated with tumor stage.

The high protein expression levels of CCNA2 in the cancerous samples were further confirmed by the results from the HPA dataset (Fig. 9). The KIF2C and BUB1B protein data were missing from the HPA dataset. Finally,

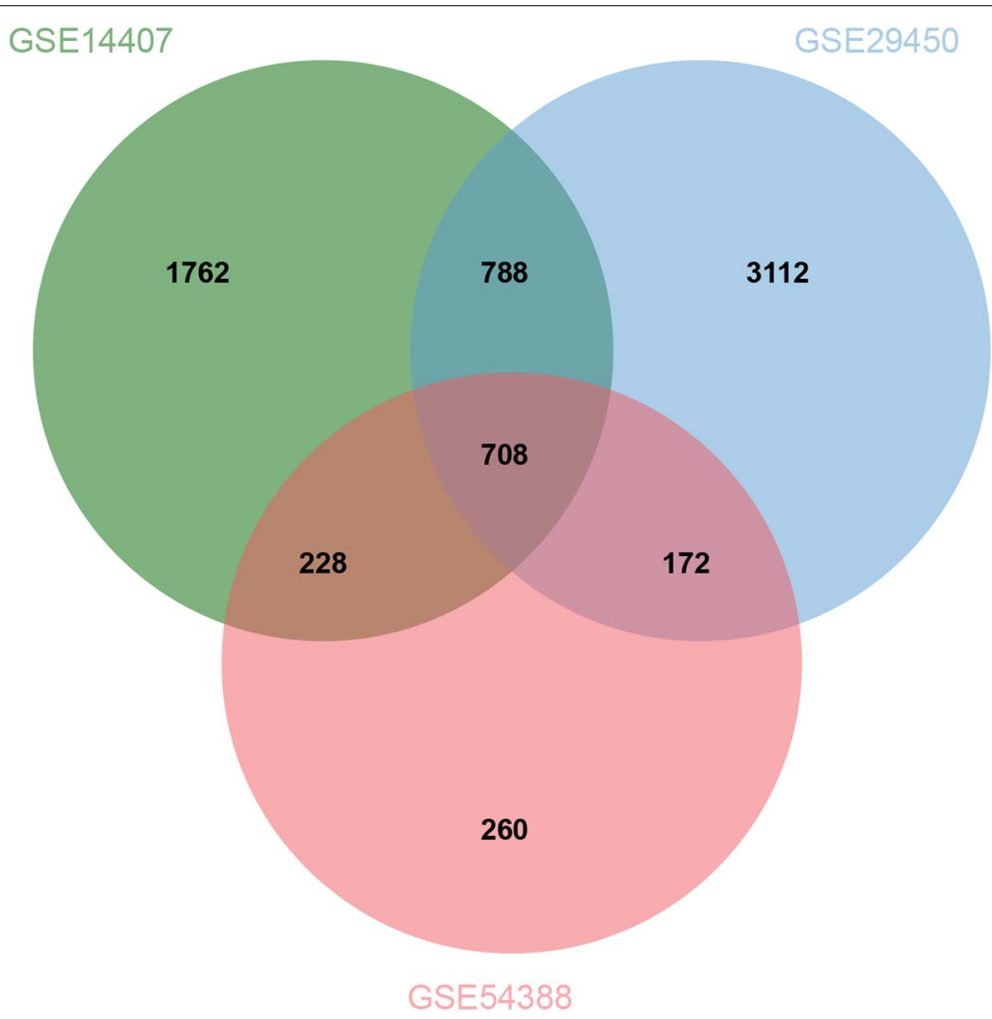

Fig. 2 Venn diagram presenting the number of overlapping genes among three GEO datasets (GSE54388, GSE14407, and GSE29450) 


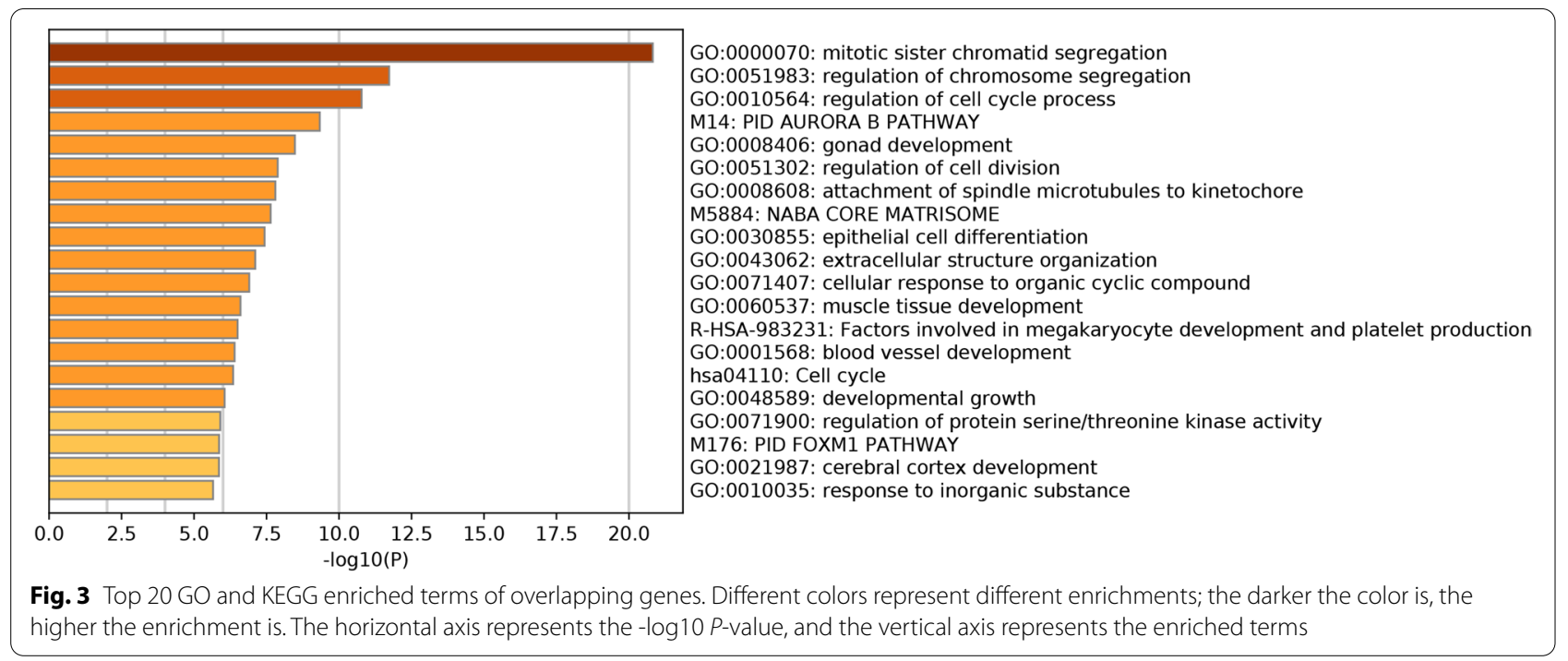

we identified CCNA2 as the hub gene that is crucial for ovarian cancer progression.

\section{Discussion}

The present study is the first to explore DEGs between ovarian cancer tissues and normal tissues with three GEO datasets. We finally determined that CCNA2 that was more highly expressed in ovarian cancer, and an increased CCNA2 RNA expression level was associated with poor PPS in all patients with ovarian cancer. These findings may contribute to the understanding of the pathogenesis of ovarian cancer and thus aid in the improvement of diagnosis, treatment and patient outcome.

To identify DEGs between ovarian cancer tissues and normal tissues, we first analyzed three GEO datasets

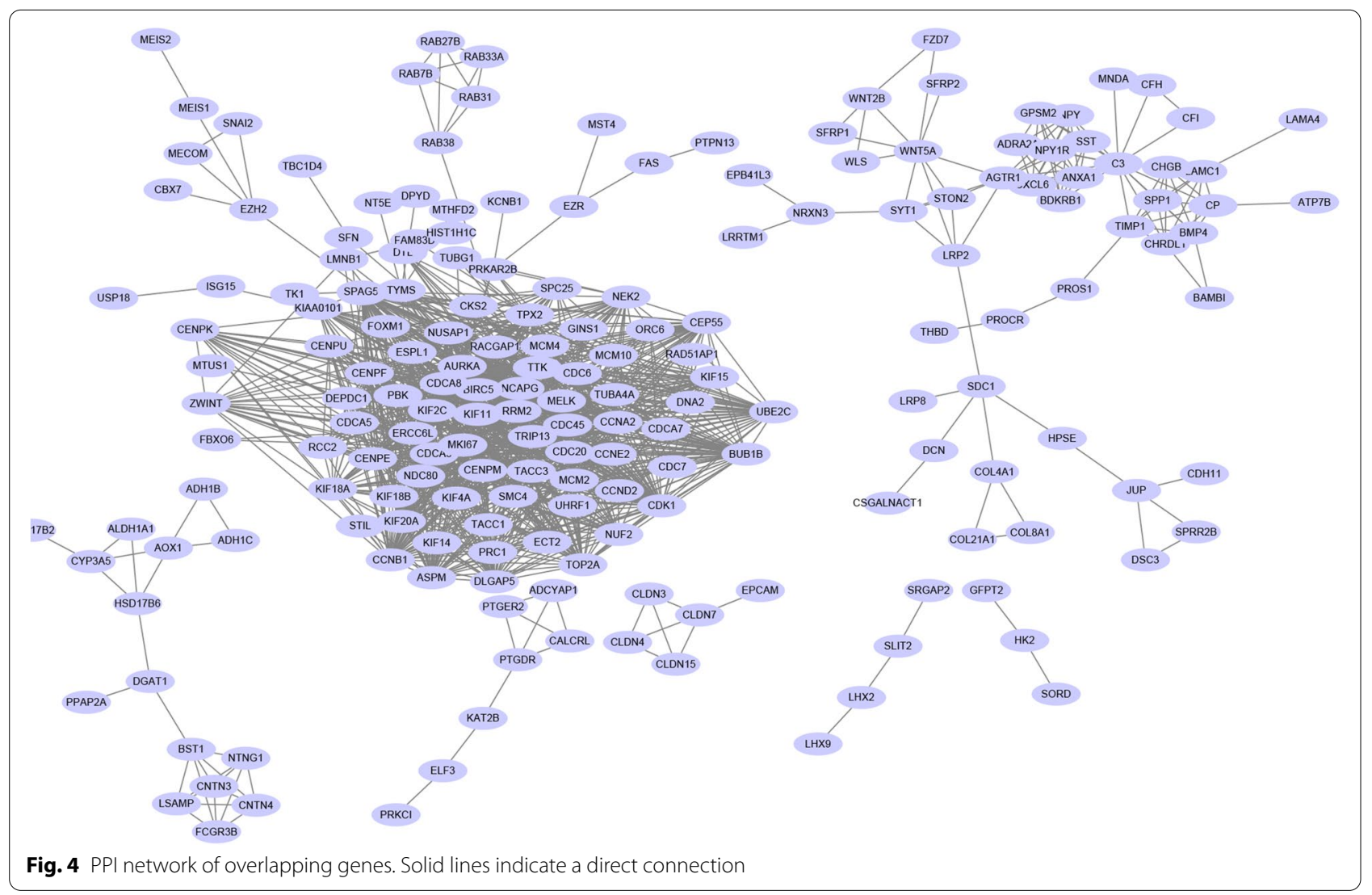




\section{MCODE model 1}

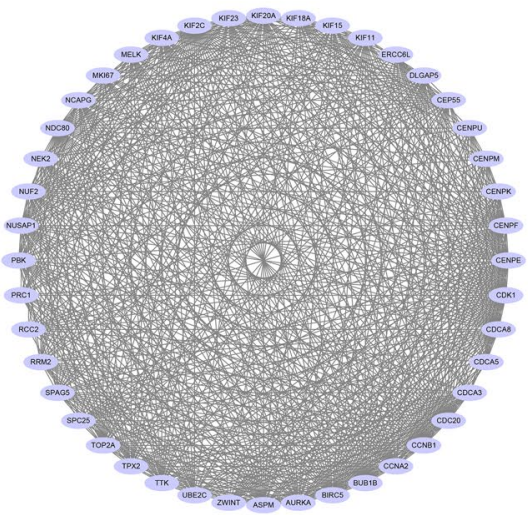

MCODE model 3

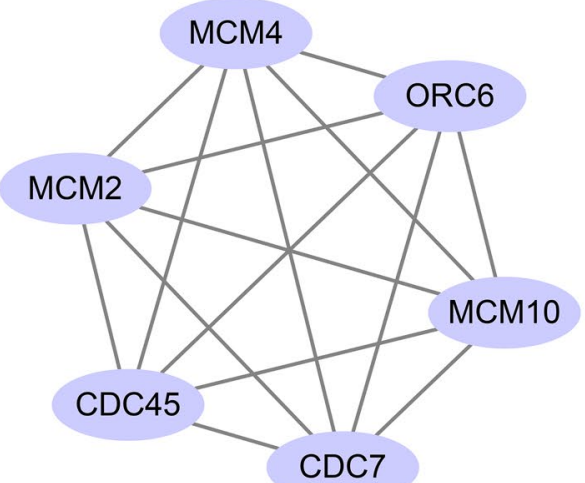

\section{MCODE model 2}

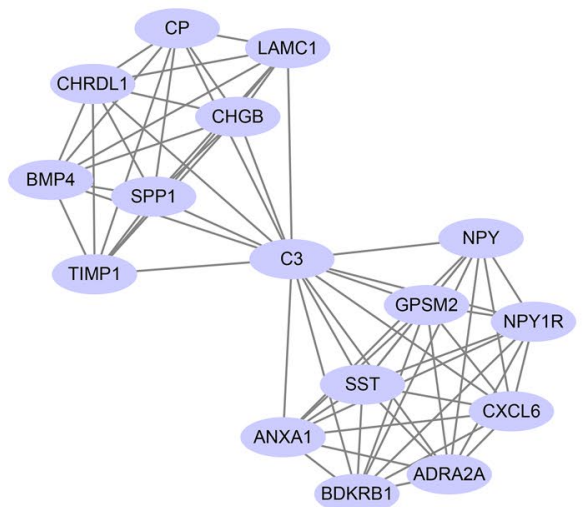

MCODE model 4

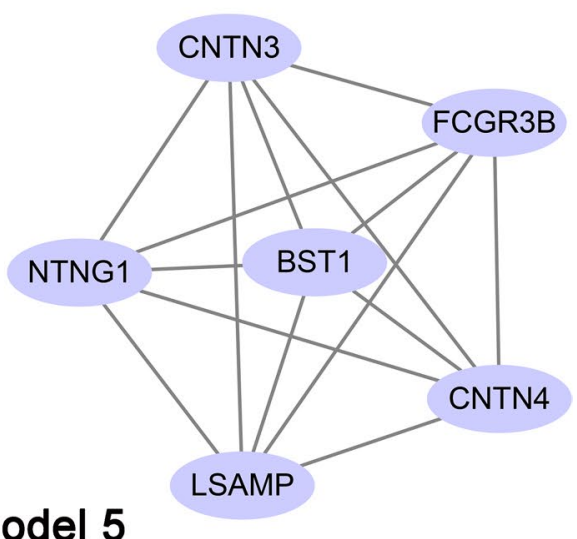

\section{MCODE model 5}

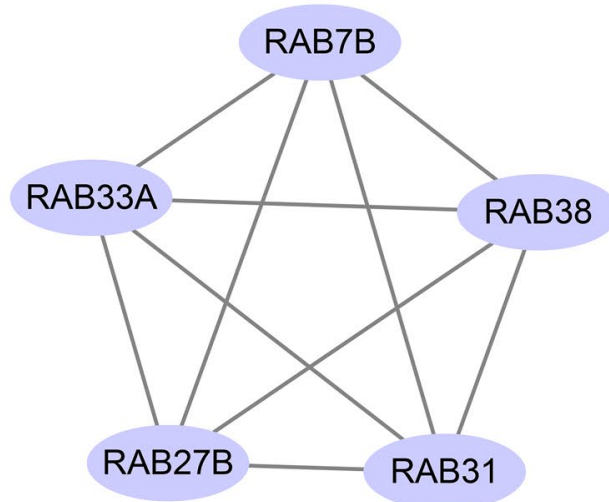

Fig. 5 Top 5 primary modules of the PPI subnetwork by plug-in MCODE in Cytoscape software

and identified 708 overlapping genes. Then, the Metascape online tool was used to further confirm the function of these genes. These DEGs were mainly enriched in the regulation of cell cycle process, cell division and blood vessel development. Finally, we identified ten hub genes (CDK1, CDC20, CCNB1, BUB1B, CCNA2, KIF11, CDCA8, KIF2C, NDC80 and TOP2A) for further analysis. Overexpression of CDK1, CCNB1 and CDC20 in tumor tissues predicted poor survival of patients with hepatocellular carcinoma [12]. The results of this study suggest that these genes are also involved in regulating the tumorigenesis and progression of hepatocellular carcinoma. However, the survival rate associated with the high expression and low expression of these 3 genes, as 


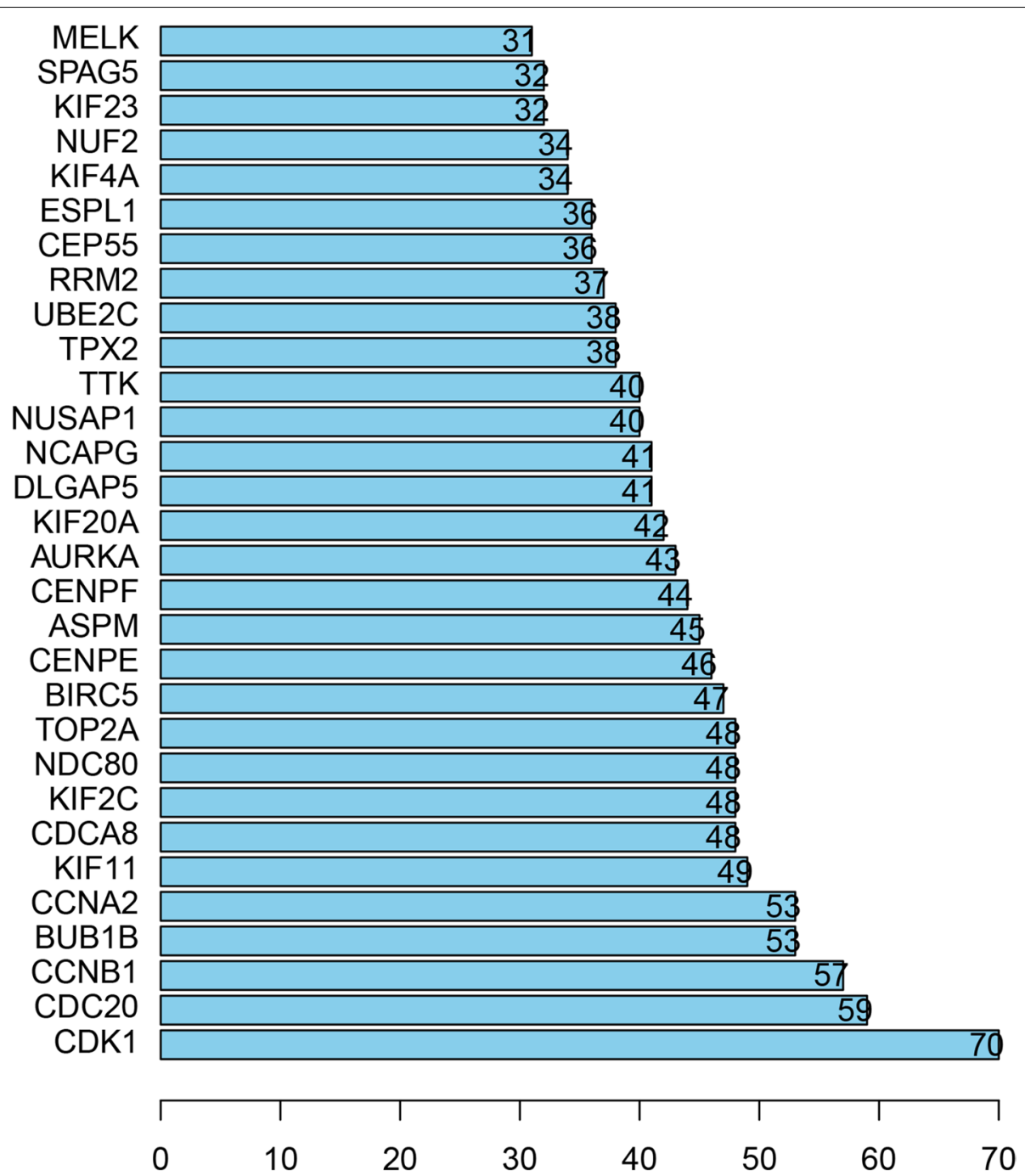

Fig. 6 The top 30 core proteins of the PPI network. The ordinate coordinates represent the name of the gene, and the abscissa represents the number of gene connections

assessed via the Kaplan-Meier method, was not significantly different.

According to Oncomine and GEPIA, these ten hub genes were more highly expressed in ovarian cancer tissues than in normal tissues. Finally, we identified CCNA2 and BUB1B as candidate hub genes for ovarian cancer progression, as their high expression was correlated with tumor stage and overall survival of ovarian cancer.

CCNA2 protein expression was further confirmed by the HPA database, which suggested that CCNA2 has potential prognostic and therapeutic significance in ovarian cancer. CCNA2 upregulation is also reportedly associated with the progression of other malignancies, including gastric cancer [13], hepatocellular carcinoma [14] and lung squamous cell carcinoma [15]. CCNA2 is a key regulatory protein that promotes the transition from $S$ phase to G2/M phase [16].

Gene ontology analysis revealed that CCNA2 was mainly enriched in mitotic sister chromatid segregation, cell division and the cell cycle. Mitotic DNA repair is thought to primarily involve sister chromatids. Abnormal mitotic DNA repair is closely related to tumorigenesis and progression [17].

The repair of DNA damage is crucial for the maintenance of genomic integrity. Cells cannot properly repair their DNA during replication without the complete set of DNA repair proteins at a damage site. In these circumstances, the outcome is mitotic catastrophe and subsequent cell death. The proteins encoded by the breast cancer gene 1/2 (BRCA1/2) genes participate in the repair of DNA double strand breaks. The loss of function 

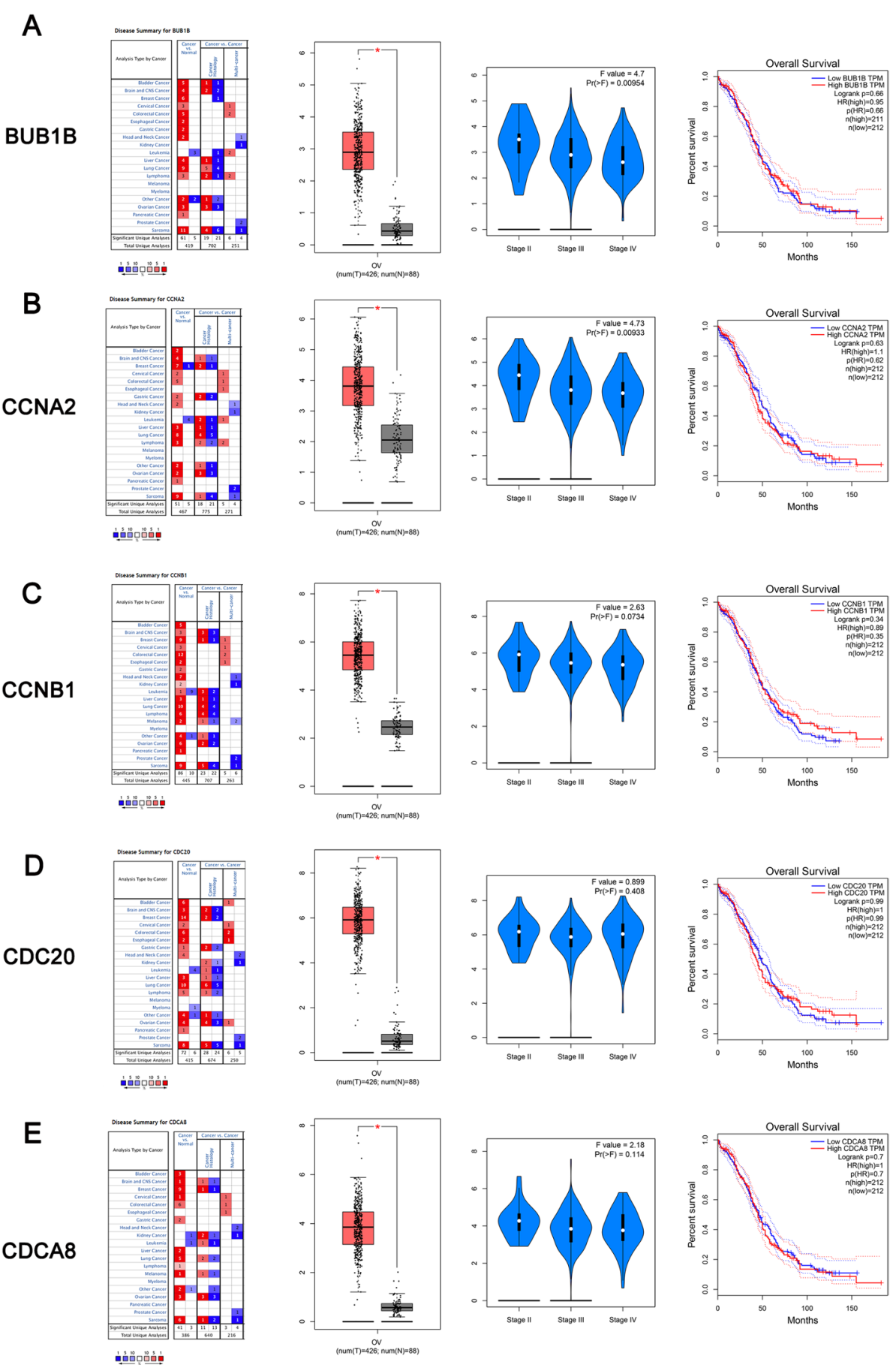

Fig. 7 A BUB1B expression and overall survival analyses in ovarian cancer tissues compared with normal tissues in the Oncomine database and GEPIA database, B CCNA2 expression and overall survival analyses in ovarian cancer tissues compared with normal tissues in the Oncomine database and GEPIA database. C CCNB1 expression and overall survival analyses in ovarian cancer tissues compared with normal tissues in the Oncomine database and GEPIA database. D CDC20 expression and overall survival analyses in ovarian cancer tissues compared with normal tissues in the Oncomine database and GEPIA database; E CDCA8 expression and overall survival analyses in ovarian cancer tissues compared with normal tissues in the Oncomine database and GEPIA database 

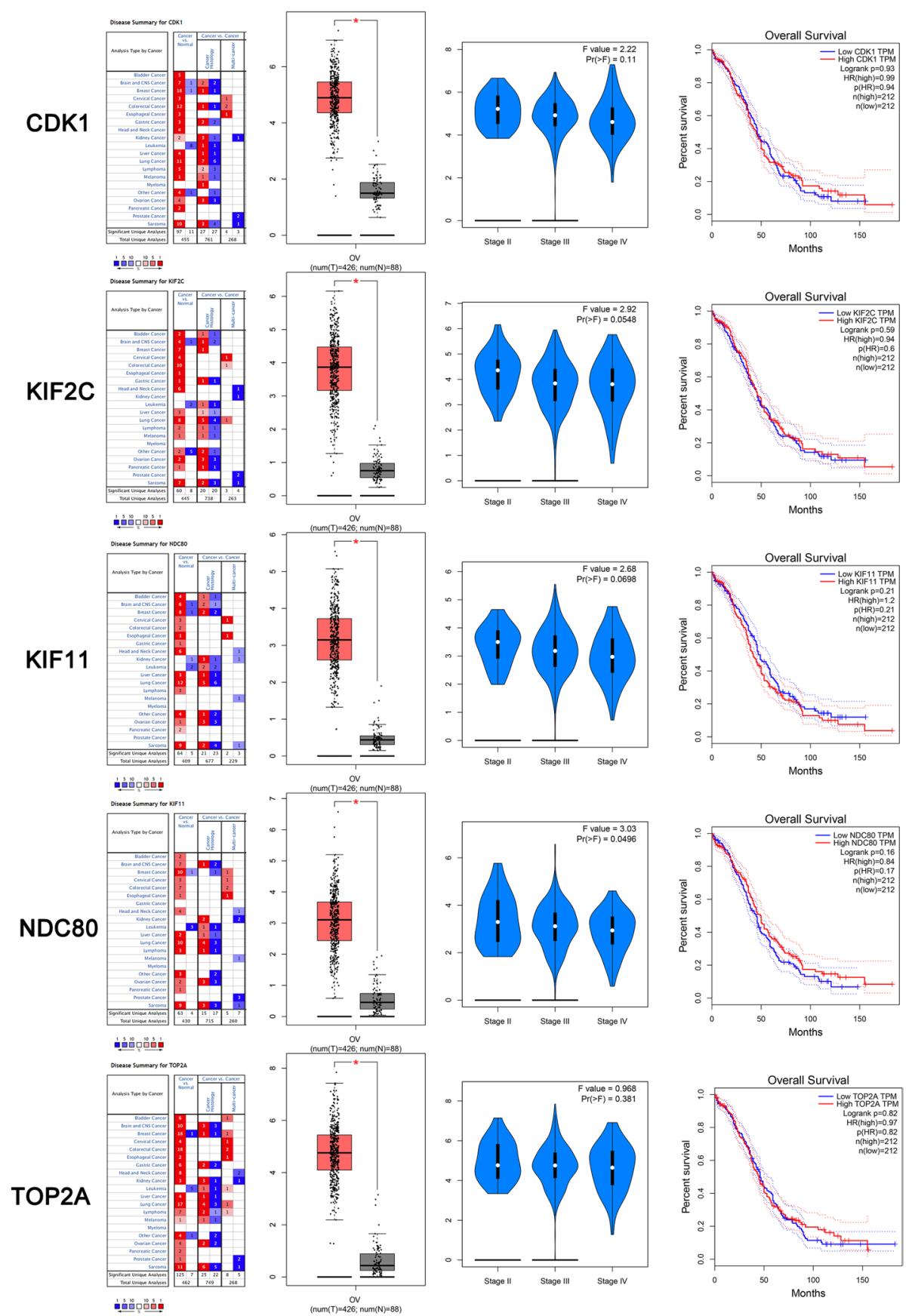

Fig. 8 CDK1 expression and overall survival analyses in ovarian cancer tissues compared with normal tissues in the Oncomine database and GEPIA database, B KIF2C expression and overall survival analyses in ovarian cancer tissues compared with normal tissues in the Oncomine database and GEPIA database. C KIF11 expression and overall survival analyses in ovarian cancer tissues compared with normal tissues in the Oncomine database and GEPIA database. D NDDC80 expression and overall survival analyses in ovarian cancer tissues compared with normal tissues in the Oncomine database and GEPIA database; E TOP2A expression and overall survival analyses in ovarian cancer tissues compared with normal tissues in the Oncomine database and GEPIA database

of these genes renders cancer cells more dependent on alternative DNA repair mechanisms, such as single-strand DNA repair. Poly(ADP-ribose) polymerase (PARP) inhibition in breast cancer mutant tumor cells induces synthetic lethality and has emerged as a promising anticancer therapy, especially in BRCA1/2 mutation carriers [18]. 


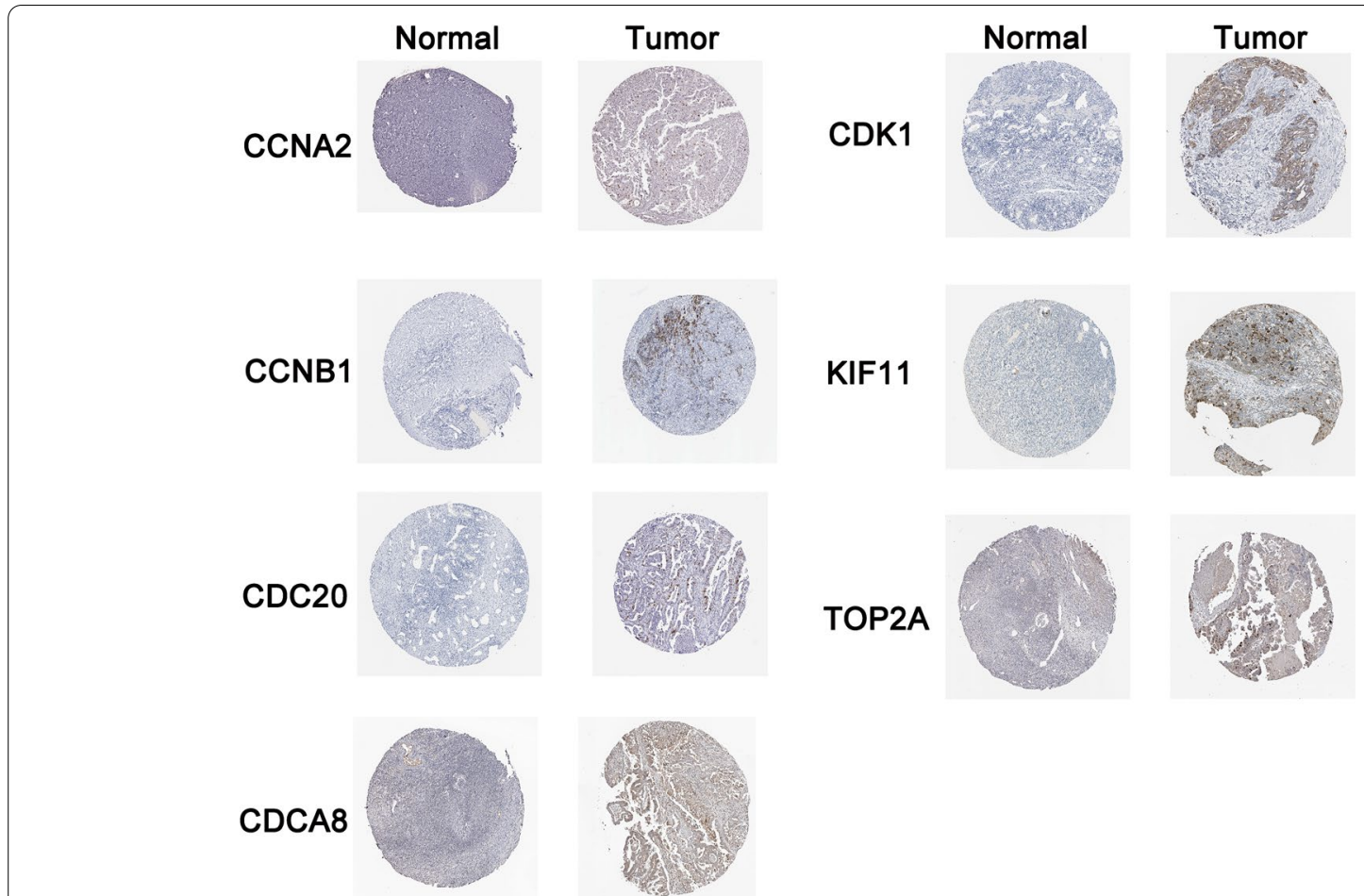

Fig. 9 Validation of the total 7 DEGs from the HPA database by immunohistochemistry

In tumorigenesis, the apoptotic cell division ratio is altered [19]. Cancer cells undergo uncontrolled cell division without programmed cell death or apoptosis [20]. Dysregulation of cell cycle progression is also considered a common characteristic of cancer [21]. CCNA2 is involved in biological processes of the cell cycle. Therefore, studies with a larger number of samples will be used to verify the results of the present study in the future.

The BUB1B gene is located at chromosome $15 \mathrm{q} 15$ and plays a vital role in chromosome segregation [22]. Ding et al. [23] revealed that BUB1B, CDK1, CCNA2, TOP2A, $\mathrm{BUB} 1 \mathrm{~B}$, and KIF11 were hub genes in the progression of colorectal cancer, and these genes were all differentially expressed in ovarian cancer. Similar findings were reported by two other research groups and showed that BUB1B participates in tumor growth and the progression of prostate cancer and lung adenocarcinoma [24, 25]. A previous study performed KEGG enrichment analysis and indicated that BUB1B was associated with the cell cycle [26]. Halting cell cycle progression is crucial for the development of tumorigenesis and tumor progression [27].

This study has some limitation. First, the pathological data were incomplete and were not included in the results of this study. Second, there was no experimental verification.

\section{Conclusion}

Taken together, our study provided evidence concerning the altered expression of genes in ovarian cancer tissues compared with normal tissues. In vivo and in vitro experiments are required to verify the results of the present study.

\section{Abbreviations}

GEO: Gene Expression Omnibus; GO: Gene Ontology; STRING: Search Tool for the Retrieval of Interacting Genes/Proteins; MCODE: Molecular complex detection; HPA: Human Protein Atlas; GEPIA: Gene Expression Profiling Interactive Analysis; OS: Overall survival; DEGs: Differentially expressed genes; PPI: Protein-protein interaction; KEGG: Kyoto Encyclopedia of Genes and Genomes.

\section{Supplementary Information}

The online version contains supplementary material available at https://doi. org/10.1186/s13048-021-00837-6.

Additional file 1: Supplement S1 Heatmap for GSE14407, GSE29450, and GSE54388.

Acknowledgements

Not applicable.

\section{Authors' contributions}

CCD and XT carried out most of the experiments and drafted the manuscript. QH, PS and XJC performed parts of the experiments and performed the statistical analysis; JYZ, PS and YMS conceived of the study and revised the manuscript. All the authors read and approved the final manuscript. 


\section{Funding}

Not applicable.

\section{Declarations}

Ethics approval and consent to participate

Not applicable.

\section{Consent for publication}

Not applicable.

\section{Competing interests}

The authors declare that they have no competing interests.

\section{Author details}

'Department of Clinical Lab, The Children's Hospital of Tianjin (Children's Hospital of Tianjin University), No. 238, Longyan Road, Beichen District, Tianjin 300000, PR China. ${ }^{2}$ Department of Medical Laboratory, The First Affiliated Hospital of Zhengzhou University, Zhengzhou, Henan Province, China.

Received: 19 March 2021 Accepted: 15 June 2021

Published online: 12 July 2021

\section{References}

1. Alexandrova E, Pecoraro G, Sellitto A. An overview of candidate therapeutic target genes in ovarian cancer. Cancers (Basel). 2020;12:1470.

2. Mahmood RD, Morgan RD, Edmondson RJ, Clamp AR, Jayson GC. Firstline management of advanced high-grade serous ovarian cancer. Curr Oncol Rep. 2020;22:64.

3. Moschetta M, Boussios S, Rassy E, Samartzis EP, Funingana G, Uccello M. Neoadjuvant treatment for newly diagnosed advanced ovarian cancer: where do we stand and where are we going? Ann Transl Med. 2020:8:1710.

4. Gorski JW, Ueland FR. CCNE1 amplification as a predictive biomarker of chemotherapy resistance in epithelial ovarian cancer. Diagnostics (Basel). 2020;10:E279.

5. Boussios S, Mikropoulos C, Samartzis E, Karihtala P, Moschetta M, Sheriff $M$, et al. Wise management of ovarian cancer: on the cutting edge. J Pers Med. 2020;10:41.

6. Zhao E, Zhou C, Chen S. Flap endonuclease 1 (FEN1) as a novel diagnostic and prognostic biomarker for gastric cancer. Clin Res Hepatol Gastroenterol. 2020:45:101455

7. Smyth GK. Linear models and empirical bayes methods for assessing differential expression in microarray experiments. Stat Appl Genet Mol Biol. 2004;3:Article3.

8. Bardou P, Mariette J, Escudié F, Djemiel C, Klopp C. jvenn: an interactive Venn diagram viewer. BMC Bioinformatics. 2014;15:293.

9. Zhou Y, Zhou B, Pache L, Chang M. Metascape provides a biologistoriented resource for the analysis of systems-level datasets. Nat Commun. 2019;10:1523.

10. Anitha P, Anbarasu A, Ramaiah S. Gene network analysis reveals the association of important functional partners involved in antibiotic resistance: a report on an important pathogenic bacterium Staphylococcus aureus. Gene. 2016;575:253-63.
11. Thul PJ, Kesson L. A subcellular map of the human proteome. Science. 2017;356:eaal3321.

12. Yang WX, Pan YY, You CG. CDK1, CCNB1, CDC20, BUB1, MAD2L1, MCM3, BUB1B, MCM2, and RFC4 may be potential therapeutic targets for hepatocellular carcinoma using integrated bioinformatic analysis. Biomed Res Int. 2019;2019:1245072.

13. Lee $Y$, Lee CE, Oh S. Pharmacogenomic analysis reveals CCNA2 as a predictive biomarker of sensitivity to Polo-Like Kinase I inhibitor in gastric cancer. Cancers (Basel). 2020;12:E1418.

14. Zeng L, Fan X, Wang X, Deng H, Zhang K, Zhang X, et al. Bioinformatics analysis based on multiple databases identifies hub genes associated with hepatocellular carcinoma. Curr Genomics. 2019;20:349-61.

15. Gao M, Kong W, Huang Z, Xie Z. Identification of key genes related to lung squamous cell carcinoma using bioinformatics analysis. Int J Mol Sci. 2020;21:2994

16. Deng J, Wang J, Khan M, Yu P, Yang F, Liang H. Structure and biological properties of five Pt (II) complexes as potential anticancer agents. J Inorg Biochem. 2018;185:10-6.

17. Ogawa S, Yamada M, Nakamura A, Sugawara T, Nakamura A, Miyajima S, et al. Zscan5b deficiency impairs DNA damage response and causes chromosomal aberrations during mitosis. Stem Cell Reports. 2019;12:1366-79.

18. Boussios S, Karihtala P, Moschetta M, Abson C, Karathanasi A, ZakynthinakisKyriakou N, et al. Veliparib in ovarian cancer: a new synthetically lethal therapeutic approach. Invest New Drugs. 2020;38:181-93.

19. Toren D, Kulaga A, Jethva M, Rubin E, Snezhkina A V, Kudryavtseva A V, et al. Gray whale transcriptome reveals longevity adaptations associated with DNA repair and ubiquitination. Aging Cell. 2020;19:e13158.

20. Dickinson K, Case A J, Kupzyk K, Saligan L. Exploring biologic correlates of cancer-related fatigue in men with prostate cancer: cell damage pathways and oxidative stress. Biol Res Nurs. 2020;20.514-9.

21. Zhu Y, Kosmacek E A, Chatterjee A, Oberley-Deegan R E. MnTE-2-PyP Suppresses prostate cancer cell growth via $\mathrm{H}(2) \mathrm{O}(2)$ production. Antioxidants (Basel). 2020;9:490.

22. Tang Q, Zhang H, Kong M, Mao X, Cao X. Hub genes and key pathways of non-small lung cancer identified using bioinformatics. Oncol Lett. 2018;16:2344-54

23. Ding $X$, Duan $\mathrm{H}$, Luo H. Identification of core gene expression signature and key pathways in colorectal cancer. Front Genet. 2020;11:45.

24. Fu X, Chen G, Cai ZD, Wang C, Liu ZZ, Lin ZY, et al. Overexpression of BUB1B contributes to progression of prostate cancer and predicts poor outcome in patients with prostate cancer. Onco Targets Ther. 2016;9:2211-20.

25. Chen H, Lee J, Kljavin NM, Haley B, Daemen A, Johnson L, et al. Requirement for BUB1B/BUBR1 in tumor progression of lung adenocarcinoma. Genes Cancer. 2015;6:106-18.

26. Duan X, Han L, Peng D, Peng C, Xiao L, Bao Q, et al. Bioinformatics analysis of a long non-coding RNA and mRNA regulation network in rats with middle cerebral artery occlusion based on RNA sequencing. Mol Med Rep. 2019;20:417-32

27. Jiang L, Wang T, Bao Y, Qian J, Wu XJ, Hu GH, et al. A study of UbcH10 expression and its association with recurrence of meningiomas. J Surg Oncol. 2012;106:327-31.

\section{Publisher's Note}

Springer Nature remains neutral with regard to jurisdictional claims in published maps and institutional affiliations. 